\title{
Observation of effect of continuous intravenous drip of low molecular dextran combined with furosemide in the treatment of edema in children with nephrotic syndrome.
}

\author{
Shipin Feng* \\ Department of Children Nephrology, Chengdu Women and Children Center Hospital, Chengdu, PR China
}

\begin{abstract}
Objective: To study the curative effect of continuous intravenous drip of low molecular dextran combined with furosemide in the treatment of edema in children with nephrotic syndrome.

Methods: A total of 170 children with edema in nephrotic syndrome treated in the Pediatric Department of our hospital from March 2015 to May 2017 were randomly divided into two groups. Patients in control group were treated with intravenous drip of furosemide (furosemide injection), while those in experimental group received intravenous drip of low molecular dextran based on the treatment in control group. The treatment indexes of the two groups were summarized.

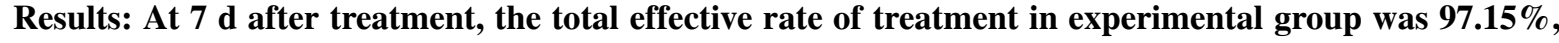
which was higher than that in control group $(88.24 \%, P<0.05)$. The extinction time of edema in experimental group was shorter than that in control group, the 24-h urine volumes at $3 \mathrm{~d}, 5 \mathrm{~d}$ and $7 \mathrm{~d}$ after treatment were larger than those in control group, and the 24-h urinary protein level was lower than that in control group $(\mathrm{P}<\mathbf{0 . 0 5})$. During the 6-month follow-up, the recurrence rate in experimental group was lower than that in control group $(\mathbf{P}<\mathbf{0 . 0 5})$.

Conclusion: Low molecular dextran combined with furosemide is significantly effective in the treatment of edema in children with nephrotic syndrome, which promotes the extinction of edema and improves the renal function, so it is worth promoting.
\end{abstract}

Keywords: Low molecular dextran, Furosemide, Edema in children with nephrotic syndrome, Urinary protein, Recurrence.

Accepted on January 27, 2018

\section{Introduction}

Nephrotic Syndrome (NS) is the clinical syndrome under the combined action of various factors, which occurs frequently in children aged 3-6 y old, especially boys, and it has a longer duration and relapses easily [1,2]. Edema is one of the four major common symptoms of children with NS, the degree of which is positively correlated with the hypoproteinemia in the body [3]. In severe edema, there are pleural effusion, ascites and scrotal edema, in addition to subcutaneous edema. The treatment is difficult and it is necessary to eliminate the edema in time and control the progression of disease [4]. In our hospital, low molecular dextran is combined with furosemide in the treatment of edema in children with NS, achieving satisfactory results. It is now reported as follows.

\section{Materials and Methods}

\section{Baseline data}

A total of 170 children with edema in nephrotic syndrome treated in the Pediatric Department of our hospital from March
2015 to May 2017 were selected, and they all met the relevant diagnostic criteria in "Clinical Diagnosis and Treatment Guidelines-Nephrology", accompanied with edema symptoms. All children had no infection and did not apply hormones recently. Children and their families volunteered to participate in the study and signed the informed consent. This research program was approved by the Ethics Committee of our hospital. Patients were randomly divided into two groups. In control group, there were 85 patients, including 61 males and 24 females aged 3-9 y old with an average of $5.21 \pm 0.76 \mathrm{y}$ old. There were 80 cases of initial onset and 5 cases of recurrence, 27 cases of mild edema, 41 cases of moderate edema, 17 cases of severe edema, 34 cases of simple limb edema, 17 cases of edema accompanied with pleural effusion, 28 cases of edema accompanied with ascites and 6 cases of edema accompanied with pleuroperitoneal fluids. In experimental group, there were 85 patients, including 64 males and 21 females aged 3-11 y old with an average of $5.30 \pm 0.79 \mathrm{y}$ old. There were 78 cases of initial onset, 7 cases of recurrence, 25 cases of mild edema, 40 cases of moderate edema, 20 cases of severe edema, 31 cases of simple limb edema, 21 cases of edema accompanied with pleural effusion, 24 cases of edema accompanied with ascites 
and 9 cases of edema accompanied with pleuroperitoneal fluids. Baseline data of child patients in the two groups were not significantly different $(\mathrm{P}>0.05)$ and were comparable.

\section{Methods}

Child patients in two groups were required to have low-salt diet, rest in bed and receive the hormones and other conventional symptomatic treatment after diagnosis. On this basis, children in control group were treated with intravenous drip of furosemide: $1 \mathrm{mg} / \mathrm{kg} . \mathrm{d}$ furosemide (furosemide injection, $2 \mathrm{~mL}$ : $20 \mathrm{mg}$ ) was given intravenously; furosemide and $50-100 \mathrm{~mL}$ saline were mixed and given intravenously once a day for $7 \mathrm{~d}$. Children in experimental group were treated with low molecular dextran on the basis of treatment of control group: $5-10 \mathrm{ml} / \mathrm{kg} . \mathrm{d}$ low molecular dextran was given intravenously once a day for $7 \mathrm{~d}$.

\section{Efficacy evaluation}

Treatment effects of child patients in the two groups were evaluated at $7 \mathrm{~d}$ after treatment. Remarkably effective: the weight of children was significantly lost, and edema in eyelids, lower extremities and other parts completely disappeared, accompanied by significant improvement in ascites and pleural effusion. Effective: the weight was lost, and edema and ascites were improved. Ineffective: no significant improvement in the disease.

\section{Observation indexes}

The following indexes were collected from the two groups: total effective rate, extinction time of edema, renal function indexes (detection of urine volume and 24-h urinary protein before treatment, and at $3 \mathrm{~d}, 5 \mathrm{~d}$ and $7 \mathrm{~d}$ after treatment), and recurrence rate during 6-month follow-up.

\section{Statistical analysis}

SPSS17.0 software was used, and enumeration data/ measurement data were compared using $\mathrm{x}^{2} / \mathrm{t}$ test; variance $\mathrm{F}$ test was used for the comparison of repeated test data. $\mathrm{P}<0.05$ suggested that the difference was statistically significant.

\section{Results}

\section{Comparison of total effective rate between two groups}

After 1 week of treatment, the total effective rate of child patients in experimental group was higher than that in control group $(\mathrm{P}<0.05)$ (Table 1).

Table 1. Total effective rates of two groups.

\begin{tabular}{lllll}
\hline Group & $\begin{array}{l}\text { Remarkably } \\
\text { effective }\end{array}$ & Effective & Ineffective & $\begin{array}{l}\text { Total } \\
\text { effective rate }\end{array}$ \\
\hline Experimental group & $43(50.59)$ & $40(47.06)$ & $2(2.35)$ & $83(97.15)^{*}$ \\
\hline Control group & $34(40.0)$ & $41(48.24)$ & $10(11.76)$ & $75(88.24)$ \\
\hline
\end{tabular}

Note: In comparison between two groups, $x^{2}=5.738 ;{ }^{*} \mathrm{P}<0.05$.

\section{Comparison of extinction time of edema between two groups}

The extinction time of edema of child patients in experimental group was shorter than that in control group $(\mathrm{P}<0.05)$ (Table 2).

Table 2. Extinction time of edema in two groups.

\begin{tabular}{ll}
\hline Group & Extinction time of edema \\
\hline Experimental group & $18.2 \pm 4.1$ \\
\hline Control group & $25.6 \pm 4.8$ \\
\hline $\mathrm{t}$ & 10.808 \\
\hline $\mathrm{P}$ & $<0.05$ \\
\hline
\end{tabular}

\section{Comparisons of renal function indexes between two groups}

The urine volume and urinary protein were not significantly different between two groups before treatment $(\mathrm{P}>0.05)$; with the advance of treatment in two groups, urine volume was increased significantly, but urinary protein was decreased significantly $(\mathrm{P}<0.05)$; the urine volumes in experimental group at $3 \mathrm{~d}, 5 \mathrm{~d}$ and $7 \mathrm{~d}$ after treatment were higher than those in control group, but the urinary protein was less than that in control group $(\mathrm{P}<0.05)$ (Table 3$)$.

Table 3. Renal function indexes in two groups.

\begin{tabular}{|c|c|c|c|}
\hline Group & Time & $\begin{array}{l}\text { Urine volume } \\
(\mathrm{ml} / 24 \mathrm{~h})\end{array}$ & $\begin{array}{l}\text { Urinary protein } \\
(\mathrm{g} / 24 \mathrm{~h})\end{array}$ \\
\hline \multirow[t]{4}{*}{ Experimental group } & Before treatment & $479 \pm 35$ & $5.87 \pm 0.94$ \\
\hline & $3 \mathrm{~d}$ after treatment & $1210 \pm 74^{*}$ & $4.32 \pm 0.55^{*}$ \\
\hline & $5 \mathrm{~d}$ after treatment & $1438 \pm 104^{*}$ & $3.10 \pm 0.31^{*}$ \\
\hline & $7 \mathrm{~d}$ after treatment & $1687 \pm 132^{*}$ & $1.26 \pm 0.21^{*}$ \\
\hline \multirow[t]{4}{*}{ Control group } & Before treatment & $477 \pm 34$ & $5.82 \pm 0.98$ \\
\hline & $3 \mathrm{~d}$ after treatment & $980 \pm 76$ & $5.11 \pm 0.68$ \\
\hline & $5 \mathrm{~d}$ after treatment & $1287 \pm 113$ & $3.79 \pm 0.38$ \\
\hline & $7 \mathrm{~d}$ after treatment & $1426 \pm 139$ & $2.18 \pm 0.26$ \\
\hline
\end{tabular}

Note: Intergroup comparison; ${ }^{*} \mathrm{P}<0.05$.

\section{Recurrence rate during 6-month follow-up}

Patients were followed up for 6 months. There was 1 case of recurrence in experimental group with the recurrence rate of $1.18 \%$; there were 8 cases of recurrence in control group with the recurrence rate of $9.41 \%\left(\mathrm{x}^{2}=5.749, \mathrm{P}<0.05\right)$. 


\section{Discussion}

NS frequently occurs in preschool and school-aged children, the majority of which is simple nephropathy, and manifested as minimal change nephrotic syndrome. Glucocorticoid is a common treatment means, and most children can be cured, but there are also some children who are insensitive to glucocorticoid, so the disease relapses and the healing is delayed, finally developing into refractory nephropathy, affecting the healthy growth and development of children $[5,6]$. Factors affecting the prognosis of NS in children include the long-term repeated edema. Due to the damage to glomerular filtration barrier and excretion of a large number of proteins via urine, the loss of a large number of urinary protein causes hypoproteinemia and the decreased plasma colloid osmotic pressure makes the water in plasma go into the tissue space through the blood vessels, leading to edema. Edema is a common symptom of NS in children, which is generally manifested as edema in eyelids and face, then gradually spreading to limbs and body. In severe cases, white and purple marks appear in thighs, medial upper arm and abdominal skin, as well as edema accompanied by decreased urine volume. Timely treatment is of significance in eliminating the edema and alleviating the pathological changes. If not treated in time, it may cause intractable edema, leading to recurrent NS.

In clinical field, diuretics have been widely used in different parts of kidney, promoting the excretion of water and electrolytes and eliminating the edema [7]. According to the mechanism, diuretics can be divided into four types: loop diuretics, thiazide diuretics, potassium-sparing diuretics and carbonic anhydrase inhibitors. Different diuretics act on different parts of renal tubules. In this study, furosemide (furosemide injection) was the loop diuretic, which is the most widely-used diuretic in clinical practice. It is released from the proximal renal tubule through the organic anion secretion pathway, reaches the lumen membrane, inhibiting the $\mathrm{Na}$ ${ }^{+}-2 \mathrm{CL}-\mathrm{K}^{+}$co-transporter on lumen membrane, thus resulting in diuretic effect. However, the long-term application of furosemide may lead to hypertrophy in distal tubule and diuretic antagonism; the application of a large amount of furosemide in a short time may break the physiological balance in children and lead to serious complications, so diuretics should be used rationally. In most NS in children, edema can be alleviated through diuretics and limiting sodium diet, but some children with severe edema suffer from serious watersodium retention. It is difficult to effectively control edema through simple diet control and diuretic treatment, so it is needed to search more effective method.

Intravenous drip of albumin can benefit the urine and sodium, but the albumin injected intravenously should be excreted via the urine, thus increasing the burden of glomerular filtration and tubule reabsorption, and also increasing the urinary protein content. A large amount of proteinuria may cause glomerular epithelial damage, so it is difficult to eliminate the edema for a long time. Moreover, albumin is expensive, also belongs to the blood product with the risk of blood-borne diseases. Low molecular dextran is the colloidal solution, as well as blood volume expander, which is used for NS in children to inhibit platelet, erythrocyte aggregation, reduce blood viscosity and inhibit coagulation factor II, preventing thrombosis. At the same time, low molecular dextran still has some osmotic diuretic effect. Intravenous drip can transiently increase the osmotic pressure of blood plasma and promote the water in tissue space to return to the blood vessel, resulting in the hypertonic state of liquid in renal tubule and reducing the water-sodium reabsorption, thus benefiting the urine and eliminating the edema [8,9]. Zhongmei studied and pointed out that compared with albumin combined with furosemide in the treatment of NS with severe edema, low molecular dextran combined with furosemide has more significant effect, which is conducive to promoting the edema extinction with a low recurrence rate [10]. Low molecular dextran combined with furosemide can exert the osmosis and diuresis effects, reduce edema symptoms, expand the renal blood vessels, reduce the renal vascular resistance and increase the renal blood flow; moreover, it can also promote the discharge of water, sodium, chlorine, potassium, calcium, magnesium and other water and electrolytes, reduce the prostaglandin synthase activity, and increase the prostaglandin E2 content, thus expanding the blood vessels. The synergistic effect of the two drugs can eliminate the edema and promote the improvement of renal function [11]. Results in this study showed that the total effective rate of child patients in experimental group was higher than that in control group, and the extinction time of edema was shorter, the urine volume was significantly higher than that in control group, and the 24-h urinary protein content was lower than that in control group, suggesting that the effect of drug combination is much better than that of simple diuretic treatment. At the same time, the recurrence rate in experimental group during follow-up was lower, and the drug combination can help improve the renal function, and avoid repeated edema and recurrence in children.

In conclusion, low molecular dextran combined with furosemide in the treatment of edema in NS children is helpful to promote the extinction of edema, control the progression of disease, improve the renal function and increase the life quality of child patients, so it is worthy of promotion and application.

\section{References}

1. Bruno G, Judith F, Pierre AC. Intra- and postoperative adverse events in children with nephrotic syndrome requiring surgery under general anesthesia. Paediatr Anaesth 2012; 22: 244-249.

2. Martha AR. The many faces of minimal change nephrotic syndrome: An overview and case study. J Am Nephrol Nurses' Assoc 2012; 39: 365-376.

3. Maeda M, Fujimoto N, Satoh T. Bilateral breast edema associated with nephrotic syndrome. Eur J Dermatol 2013; 23: 739-740.

4. de Benedetto U, Pastore MR, Parodi MB. Retinal involvement in nephrotic syndrome secondary to minimal change disease. Eur J Ophthalmol 2012; 22: 843-845. 
5. Sauter M, Berger F, Fischereder M. Nephrotic syndrome in a woman with tuberous sclerosis. Deutsche Medizinische Wochenschrift 2014; 139: 1046-1050.

6. Abassi Z, Weissman I, Karram T. Restoration of renal responsiveness to atrial natriuretic peptide in experimental nephrotic syndrome by albumin infusion. Am J Nephrol 2013; 38: 292-299.

7. Miyamoto T, Ishikawa Y, Yamamoto J. Thrombotic microangiopathy secondary to steroid pulse therapy administered for refractory nephrotic syndrome. Intern Med 2013; 52: 2099-2103.

8. Al-Hamed MH, Al-Sabban E, Al-Mojalli H. A molecular genetic analysis of childhood nephrotic syndrome in a cohort of Saudi Arabian families. J Hum Genet 2013; 58: 480-489.

9. Ueda K, Hirahashi J, Seki G. Successful treatment of acute kidney injury in patients with idiopathic nephrotic syndrome using human atrial natriuretic peptide. Intern Med 2014; 53: 865-869.
10. Li ZM. Comparison of effects of different drug therapies in the treatment of severe edema in children with nephrotic syndrome. Contemporary Med 2011; 17: 63-64.

11. Camille M, Sumant SC. Nephrotic syndrome: components, connections, and angiopoietin-like 4-related therapeutics. J Am Soc Nephrol 2014; 25: 2393-2398.

\section{*Correspondence to}

Shipin Feng

Department of Children Nephrology

Chengdu Women and Children Center Hospital

Chengdu

PR China 